\title{
Ce qu'on a appelé « néoréalisme cinématographique »
}

Di ciò che è stato chiamato «neorealismo cinematografico»

Pierre Sorlin

\section{CpenEdition}

\section{Journals}

Édition électronique

URL : http://journals.openedition.org/cei/5490

DOI : $10.4000 /$ cei.5490

ISSN : 2260-779X

Éditeur

UGA Éditions/Université Grenoble Alpes

Édition imprimée

ISBN : 978-2-37747-076-1

ISSN : 1770-9571

Référence électronique

Pierre Sorlin, « Ce qu'on a appelé « néoréalisme cinématographique » », Cahiers d'études italiennes [En ligne], 28 | 2019, mis en ligne le 15 février 2019, consulté le 26 mars 2021. URL : http:// journals.openedition.org/cei/5490 ; DOI : https://doi.org/10.4000/cei.5490

Ce document a été généré automatiquement le 26 mars 2021.

(C) ELLUG 


\title{
Ce qu’on a appelé « néoréalisme cinématographique »
}

\author{
Di ciò che è stato chiamato «neorealismo cinematografico»
}

\author{
Pierre Sorlin
}

1 Pendant la seconde moitié $\mathrm{du} \mathrm{xx}^{\mathrm{e}}$ siècle les réalisateurs italiens et, hors de la péninsule, la Nouvelle Vague française, le Cinema Novo brésilien, la Nowa Fala polonaise, la Nová via tchèque se sont tous réclamés du néoréalisme pour affirmer leur volonté d'en finir avec les « recettes de papa » et rendre aux films une dynamique perdue. Leur objectif n'était ni d'imiter Rossellini ou Visconti, ni de faire « mieux », mais de se situer par rapport à une manière originale de filmer. En tant que moment fondateur, le néoréalisme italien a été une référence fondamentale pour le cinéma pendant le second $\mathrm{xx}^{\mathrm{e}}$ siècle.

$2 \mathrm{Au} \mathrm{xxI}{ }^{\mathrm{e}}$ siècle, les repères ont changé, couleur, traitement digital des plans et du montage ont renvoyé au passé noir et blanc et prise de vue directe et, désormais, les films asiatiques attirent davantage que les productions européennes. Cités par les histoires du cinéma, rarement projetés, les films néoréalistes semblent appartenir au passé, le temps est venu de les revisiter en les prenant tels que les voyait leur époque.

Commodes pour désigner un ensemble d'œuvres, les étiquettes sont également trompeuses, elles suggèrent en un mot trop de choses qu'elles ne définissent pas. « Néoréalisme » implique un autre réalisme, antérieur, qu'on entend revivifier. À toutes les époques on débat du réalisme, terme valise, utile parce qu'on peut l'entendre de cent manières différentes. Au cours des années trente, en Italie, les tenants du régime réclamaient la mise en œuvre d'un réalisme authentiquement fasciste, leurs adversaires plaidaient pour un réalisme sincère, capable de dévoiler les faux-semblants $\mathrm{du}$ fascisme. De part et d'autre on cherchait un nouveau réalisme, la presse évoquait, sans chercher à le caractériser, le "Neorealismo" - j'ai trouvé dans l'Almanacco letterario pour 1930 un portrait de l'écrivain moderne doté d'un "occhio neorealista ", d'autres ont repéré le terme dès 1927, peu importe, il circulait largement. Appliqué, au lendemain de la guerre à certains romans, il déborda vers le cinéma, grâce notamment à Guido Aristarco, rédacteur en chef de la revue Cinema, qui l'appliqua à des films soucieux des problèmes sociaux de l'époque, dont l'intrigue ne s'attardait pas sur des 
drames personnels et refusait tout sentimentalisme. L'étiquette ne faisait d'ailleurs pas l'unanimité ; un autre journaliste, Fernaldo Di Giammatteo, ironisait dans Bianco e Nero, revue du Centro Sperimentale della Cinematografia, sur « certa arida, inconcludente e disperata pseudo poetica che va da qualche anno sotto il nome di "neorealismo"1 ".

\section{Quels films?}

4 Les « ...ismes », futurisme, surréalisme, suprématisme, se sont construits autour d'un manifeste auquel des artistes ont adhéré. Le néoréalisme filmique n'a été ni un mouvement ni une école et n'a jamais eu de programme. Au début des années cinquante il est apparu comme une évidence bien que personne ne s'en réclame; l'adjectif a été accolé, sans raison précise, à des films extrêmement différents de sorte que, aujourd'hui encore, la liste des œuvres qui méritent ce titre fluctue, selon les auteurs, de quelques réalisations à plus d'une trentaine. Je propose de nous en tenir aux sept réalisations à propos desquelles existe un consensus, chacun étant libre d'y ajouter d'autres titres. Ce sont :

- Roma città aperta (1945), scénario : Sergio Amidei ; réalisation: Roberto Rossellini ; chef opérateur : Ubaldo Arata.

- Paisà (1946), scénario : Sergio Amidei ; réalisation : Roberto Rossellini ; chef opérateur : Otello Martelli.

- Sciuscià (1946), scénario : Cesare Zavattini, Sergio Amidei ; réalisation : Vittorio De Sica ; chef opérateur : Anchise Brizzi.

- Germania anno zero (1947), scénario et réalisation: Roberto Rossellini ; chef opérateur : Robert Julliard.

- Ladri di biciclette (1947), scénario : Cesare Zavattini ; réalisation: Vittorio De Sica ; chef opérateur : Carlo Montuori.

- La terra trema (1947), scénario et réalisation : Luchino Visconti ; chef opérateur : Graziati Aldo.

- Umberto D. (1951), scénario : Cesare Zavattini ; réalisation : Vittorio De Sica ; chef opérateur : Graziati Aldo.

5 Une mauvaise habitude, héritée des études littéraires, consiste à traiter comme «auteur » le réalisateur d'un film. Ce dernier a un rôle majeur, mais la réalisation est une œuvre collective; le metteur en scène expose sa conception, du sujet, des images, de leur enchaînement, le chef opérateur choisit, en dernière analyse, éclairage, cadrage, format, angles de prise de vue, le monteur gère enchaînements et transitions. On remarque, dans la liste précédente, des « couples ", Rossellini et Amidei, De Sica et Zavattini ; le même technicien, Eraldo Da Roma a monté Roma città aperta, Paisà, Sciuscià, Germania anno zero, Umberto $D$.; Visconti avait déjà travaillé avec Mario Serandrei, monteur de La terra trema. Ces films ont été réalisés par un petit groupe de cinéastes et de techniciens intéressés par l'expérience, acceptant donc d'être mal payés. Il faudrait bien entendu tenir compte également des acteurs, mais c'est une autre question qui reviendra plus loin.

6 À la liste communément admise on ajoute parfois Caccia tragica ${ }^{2}$, Riso amaro ${ }^{3}$, Il cammino della speranza $a^{4}$ et quelques autres films moins importants. Le choix n'est pas indifférent, il traduit une conception du néoréalisme. Ces films ont en commun une certaine attention à des problèmes qui se posaient de façon urgente, en Italie, au lendemain de la guerre: reconstruction, prise en charge des personnes déplacées et des militaires 
démobilisés, surpopulation du Mezzogiorno, émigration forcée vers des pays riches. Pour tout un courant critique le principal caractère du néoréalisme est un regard sans complaisance sur un pays ruiné par le conflit, divisé, miné par les inégalités entre régions et entre classes sociales. Légitime dans la mesure où le néoréalisme n'a jamais été défini, une telle lecture se heurte à de nombreuses difficultés.

Guido Aristarco avait noté dès 1949 " Gli equivoci sul neorealismo ${ }^{5}$ » qu'on réduit trop souvent, écrivait-il, à « una verità che si basa sull'autenticità dei luoghi ove l'azione si svolge e su una vicenda "ispirata" a fatti accaduti » et même, au pire, à «il colore, la macchietta, il dialetto » quand il s'agit en fait d'un profond remaniement stylistique et narratif. Prenons le film le plus souvent mentionné par ceux qui défendent ce point de vue, Riso amaro. Trois séquences de type documentaire, réparties au long de la projection, y évoquent le sort des mondine arrivant de toute l'Italie, replantant des pousses de riz, les pieds dans l'eau, le dos courbé, surveillées sous un soleil de plomb par des contremaîtres impitoyables, parquées dans des dortoirs, repartant avec, comme salaire, un sac de riz. Vingt minutes d'enquête sans concession contre une heure vingt de mélodrame: deux couples, un gangster et une allumeuse d'une part, un brave sergent et une voleuse repentie de l'autre, s'affrontent ; leur duel finit dans un abattoir où le méchant est tué, la perverse se suicide, les bons partent joyeusement - autrement dit un scénario hollywoodien émaillé de sérieuses informations critiques ${ }^{6}$.

De l'après-guerre aux années soixante la dénonciation des injustices est apparue, au moins en arrière-plan, dans un bon tiers des films. Inversement, elle n'était pas dominante dans les sept réalisations que nous avons isolées; Roma città aperta et Paisà traitent de la guerre, Germania anno zero de l'Allemagne ruinée et affamée par le conflit, La terra trema et Umberto $D$. abordent des questions, soumission des pêcheurs siciliens à l'arbitraire des mareyeurs ${ }^{7}$, isolement des retraités sans famille, qui ne sont pas particulières aux années quarante. Seuls Sciuscià - les orphelins victimes des hostilités - et Ladri di biciclette - le chômage des travailleurs sans qualification - se rapportent directement à l'époque de la reconstruction. Chercher, à travers le néoréalisme, un regard sur une Italie déboussolée, marquée par la pénurie, les destructions, l'incertitude politique semble donc réducteur ; les réalisations où l'on fait allusion au présent sont trop nombreuses pour former un ensemble un tant soit peu cohérent, celles que nous avons retenues parce qu'elles font l'unanimité vont bien audelà d'un simple constat.

9 À côté de l'attention prêtée aux désastres du présent, on mentionne souvent, comme traits spécifiques du néoréalisme, le remplacement des acteurs professionnels par de simples citoyens et le tournage en décors naturels. On oublie ainsi que le film qui, pour tout le monde, fixe le début du néoréalisme, Roma città aperta, a pour protagonistes trois vedettes, Anna Magnani, Aldo Fabrizi, Marcello Pagliero et qu'il fut presque entièrement tourné en studio. Seule La terra trema remplit les deux critères que nous avons cités. Les protagonistes des autres films sont bien des amateurs, mais ils sont entourés de professionnels, leurs voix sont doublées par des acteurs et les scènes d'intérieur ont été faites en studio. La présence de Lamberto Maggioranti, ouvrier à la Fiat dans Ladri di biciclette, celle de Carlo Battisti, professeur de philologie incarnant Umberto $D$. donnent incontestablement une tournure très particulière à leur rôle ; leurs gestes peu calculés, parfois un peu gauches enlèvent à leurs personnages la «justesse » dont des professionnels auraient fait preuve, la rudesse, les mouvements parfois brutaux des marins et de leurs proches distinguent La terra trema de tout autre 
film. S'ils sont l'un des atouts du néoréalisme, les amateurs sont très loin de constituer l'ensemble des castings.

\section{Un nouveau cinéma}

10 structures narratives. Le cinéma, à ses débuts, a transcrit pour l'écran beaucoup de
romans, la logique romanesque, linéaire et chronologiquement ordonnée, est devenue peu à peu le moule d'une grande majorité de scénarios: une situation initiale détermine une crise dont les développements constituent le corps de la réalisation et qui se dénoue, généralement bien, parfois mal, mais toujours de façon claire. L'immense succès du cinéma, qui fut le divertissement public le plus fréquenté des années vingt aux années soixante-dix résulta, pour une large part, de cette constante ; les spectateurs connaissaient les règles de base, ils se demandaient seulement de quelle façon, à chaque fois, elles prendraient forme. Les œuvres néoréalistes rompent avec ce canevas où les épisodes se commandent les uns les autres, elles proposent différents déroulements, tous originaux et libérés de l'inférence logique; je ne peux pas les énumérer tous, je me limiterai à quatre cas singuliers et novateurs.

11 Roma città aperta construit une seule histoire (activités et défaite d'un réseau de partisans dans la ville occupée par les Allemands) en opposant stylistiquement et émotionnellement deux traitements filmiques. La première moitié du film se déroule au milieu du popolo minuto capable, grâce à une profonde solidarité, de supporter rationnement et répression policière. On est ici dans le registre des « commedie alla romana » où les mêmes Anna Magnani et Aldo Fabrizi faisaient preuve de patience et de débrouillardise. Avec l'occupation les choses ont empiré, mais courage et solidarité demeurent. La transition vers la seconde partie du film est extrêmement audacieuse. Dans une scène burlesque le prêtre, pour échapper aux soupçons des fascistes, assomme un vieillard avec une poêle à frire ; on rit et soudain, en quelques secondes, les Allemands tirent, Pina, l'héroïne est tuée sous les yeux de son fils, le prêtre, réalisant une Pietà inversée, prend sur ses genoux le corps de la morte. On entre alors en pleine tragédie sur une tonalité quasi mystique. Le chef de la résistance est torturé à mort ; pour la première fois à l'écran on voit les pinces servant à arracher les ongles, le chalumeau, les chairs brûlées et boursouflées du partisan. Battu, flagellé, celui-ci devient un «Christ aux outrages» d'autant plus que ses cheveux épars semblent une couronne d'épines ; les Allemands, au fond, rappellent les soldats romains et l'Église, en la personne du prêtre, s'agenouille aux pieds du martyre. Le sacrifice s'achève avec l'exécution du prêtre, les enfants, revenant à Rome après avoir assisté à la mort de leur parroco, aperçoivent au loin la coupole de saint Pierre. La communion des saints, union des croyants à la divinité, s'est réalisée par le sacrifice des justes, le flambeau passe aux jeunes.

12 De nombreux films italiens ont évoqué, sur un mode généralement triomphaliste, la résistance au nazi-fascisme ; aucun n'a osé passer aussi brutalement du supportable à l'horreur, ni faire des Italiens un peuple martyr, fort seulement de sa confiance en Dieu. Roma città aperta demeure une œuvre unique, difficilement classable, superficiellement "réaliste " par son ancrage dans un quartier ouvrier de la capitale, profondément nouvelle et audacieuse par son dépassement du récit linéaire au profit d'un imprévisible et provoquant changement de régime. Son rapport aux autres films 
néoréalistes est incertain, pour ne pas dire inexistant, mais il a sa place parmi les réalisations innovantes des années quarante comme manifeste d'un cinéma libéré des intrigues bien construites et résolu à faire jouer avant tout l'émotion.

Si on en résume l'intrigue, Ladri di biciclette semble une histoire simple, continue, expédiée en trois jours seulement. Un chômeur obtient une place de colleur d'affiches à condition de se déplacer à bicyclette, on lui vole son vélo, il tente en vain de retrouver son voleur, ne réussit pas à dérober une bicyclette, repart chez lui humilié et bredouille. Une anecdote aussi sobre autorise à parier sur le temps, chaque épisode, au lieu de conduire mécaniquement à l'étape suivante, est exploré pour lui-même ; deux regards interviennent simultanément, celui du chômeur, obnubilé par son vélo, insensible aux lieux qu'il traverse, celui du spectateur flânant tour à tour à travers un marché sur une place publique, dans une église de l'île Tibérine où des clochards doivent assister à la messe pour recevoir une soupe, dans une maison de passe, une trattoria, un logement ouvrier, avant de se mêler à une sortie de match. C'est un dimanche, les gens se promènent, le ciel s'obscurcit, il pleut, on se sèche, le soleil réapparaît, l'homme se hâte, le public se distrait. Et l'histoire n'a pas de fin, que se passera-t-il ensuite? Parviendra-t-il à emprunter une bécane? Retournera-t-il au chômage? Touchante ou angoissante la fin qui pourrait clore ce mini drame est esquivée, le sujet était une double errance dans Rome, non un prétexte à attendrissement.

Le chômeur sans bicyclette poursuit un objectif. Umberto D., s'il parcourt également les rues de la capitale, n'a aucun but. Il fait de maladroites tentatives pour améliorer un peu sa pension, sans prendre garde à ce qui l'entoure, ni remarquer combien, la reconstruction terminée, Rome est en train de changer rapidement. Sur ses pas, de nouveau, le spectateur aperçoit, lui, l'agitation d'un jour de semaine, une cantine populaire, un immeuble en réfection, un jardin public, il se fait complice du vieil homme qui observe un couple clandestin, bavarde avec la bonne de sa logeuse, entre à l'hôpital pour gagner quelques repas gratuits. Le séjour dans la salle commune marque un tournant du film, désormais Umberto regarde, le public voit et entend avec lui les travaux qui vont faire d'une zone longtemps périphérique un quartier bourgeois, la vie des rues, le bavardage des passants, le désarroi de la domestique enceinte dont le vieil homme tente de raisonner le séducteur. La propriétaire chasse le retraité qui ne lui rapporte pas assez, Umberto est dehors, sans rien, il pense au suicide mais il a son chien, le film le quitte tandis qu'il s'amuse joyeusement avec l'animal. Aucun pathos, une fin souriante après des jours gris, on est dans la fiction même si les images de Rome et de ses habitants ont été prises sur le vif.

Ni le chômeur de Ladri di biciclette ni Umberto ne sont les protagonistes de deux histoires qu'ils ne font pas avancer, ils servent plutôt de passeurs, entraînant le public, lui laissant le choix entre s'apitoyer sur la détresse des personnages ou découvrir les cent facettes offertes par une ville à qui la traverse. Démarche nouvelle où une mince intrigue se met en route, avance lentement, ne trouve aucune conclusion. Réalisme ? Oui, si l'on considère que la majorité des humains parcourent le monde sans le voir et que, pour lui prêter attention, la meilleure solution est d'observer son reflet au cinéma.

La terra trema propose encore une autre rupture avec le cinéma dominant. La trame narrative est toujours aussi mince, un pêcheur sicilien au service d'un patron, tente de se rendre indépendant, il échoue et retourne à son ancienne situation. La minceur du sujet est compensée par le travail sur la durée, non pas une durée subjective, vécue par 
les pêcheurs et leur entourage mais une durée filmique fondée sur l'ampleur accordée aux plans. Ni raccourcis, ni ellipses, les barques rentrant au port, les âpres discussions entre pêcheurs et mareyeurs, les scènes domestiques, les dialogues résistent à la hâte, au besoin de simplifier pour mieux faire comprendre. Les silences, l'immobilité, les déplacements suivis sur toute leur trajectoire proposent au spectateur un tempo inhabituel : la liberté de regarder au lieu d'anticiper les événements et de remarquer ce que, d'ordinaire, il n'a pas le loisir de prendre en compte, la construction des images, les subtiles variations d'éclairage, le soin apporté à la mise en cadre de chaque individu. Antonioni le soulignait : « In nessune altro film italiano si incontra una tale invenzione tecnica, un così moderno uso della cinepresa, dagli effetti pan-focali ai lunghi movimenti di macchina, dalla inquadratura composta armonicamente alla fotografia lucida e incisiva ${ }^{8}$. " C'est ce que, à l'époque, on appelait du "cinéma pur ", l'agrément sensuel que proposent des images déployant, à travers leur transformation, les subtilités de la prise de vue. Toute cette sophistication pour montrer comment dans la Sicile de 1947 un pêcheur n'avait aucune chance d'échapper à sa condition? Il y a en fait, dans cette histoire très simple, un autre point de vue qui rapproche ce film des autres réalisations dont nous nous occupons.

\section{Un cinema corale}

17 De nombreux films italiens, dans la décennie qui suivit la guerre, dénoncèrent la misère, le chômage, l'exploitation des paysans et des ouvriers. Il cammino della speranza en offre un excellent exemple, la mine qui faisait vivre tout un village sicilien ferme, des familles entières sont menacées de famine, un homme propose d'émigrer en France où l'on trouve des emplois, il convainc quelques personnes de le suivre, réussit à déjouer les pièges et les trahisons et, au risque de sa vie, conduit ses compagnons jusqu'aux portes de l'espérance. Un individu, acteur connu, autour de lui une troupe : c'est ce qu'évite le néoréalisme, dans cette poignée de films individus, événements, objets échappent au héros tout puissant, les histoires tirent leur cohérence d'une action collective. L'intraduisible corale est le seul mot qui convienne pour caractériser des œuvres où une communauté se fait agent médiateur pour régler les oppositions et les conflits inhérents à toute fiction et surtout pour donner leur sens aux films.

D'une manière générale c'est une communauté qui apparaît d'abord à l'écran, un personnage est ensuite distingué, plus précisément suscité par le groupe. La terra trema s'ouvre, à l'aube, par un large panorama sur la mer filmée du rivage. Aucun mouvement interne n'anime le plan, mais des cris rythmés, monotones, introduisent une dense et invisible présence; on le comprendra peu après, les pêcheurs lancent des appels sonores pour résister à la fatigue et, dans la lumière hésitante, garder le contact entre eux, les sons psalmodiés servent à alléger collectivement une tâche difficile. Prolongée, l'image vide s'éclaire peu à peu, des barques de pêche se dessinent à l'horizon, s'approchent, accostent, les pêcheurs débarquent leurs prises et commencent à marchander avec les mareyeurs. La séquence est interrompue par une visite à une maison où les femmes préparent un repas pour le retour des hommes. Un cadrage serré, une lumière tamisée, distinguant extérieur et intérieur, soulignent la différence opposant l'espace extérieur, sphère de la communauté de travail, au domaine privé de la famille. Une troisième séquence se raccorde à la première mais, cette fois, les tractations avec les grossistes tournent au conflit, un jeune homme, montant sur une 
pierre pour se faire entendre, exhorte ses camarades à ne pas accepter des prix de misère, il se fait la voix du groupe.

Ladri di biciclette et Umberto D. commencent avec des séquences comparables. Le premier film débute dans un quartier périphérique de Rome. Un bus arrive, les passagers descendent et rejoignent d'autres personnes au pied d'un immeuble. Un fonctionnaire lit un catalogue d'emplois à pourvoir. Entendant qu'un poste de colleur d'affiches est libre, un homme court chercher un camarade, le met au courant et le tire littéralement de la foule pour le conduire au fonctionnaire. Avec Umberto $D$., c'est une foule de retraités qui manifeste dans les rues de la capitale pour exiger une augmentation des pensions et, quand la police intervient, Umberto se détache du groupe.

Ces films mettent en scène des communautés d'individus engagés dans un travail ou la recherche d'un meilleur sort, le problème de la survie matérielle crée un lien et distingue les membres d'une certaine communauté de tous ceux qui ne partagent pas les mêmes soucis. Le sentiment collectif est plus exigeant que l'affection familiale, protectrice mais incapable de donner le moyen de vivre, il est aussi plus fort que le rapport avec cette vague entité qu'on nomme société, incapable de résoudre les problèmes économiques. Les communautés représentées par ces films ne sont pas un simple arrière-plan, leur rôle dans le déroulement de l'intrigue est plus important que celui des personnages caractérisés individuellement.

Quand Roma città aperta fut tourné, durant l'hiver 1944, le nord de la péninsule était encore occupé par les Allemands. La période du néoréalisme coïncida avec la fin de la guerre et avec la périlleuse époque du retour à la normale, les films portent trace des craintes largement partagées touchant à l'avenir du pays. Pendant les derniers mois du conflit beaucoup d'Italiens, traités en ennemis et en inférieurs par les Allemands, s'étaient sentis membres d'une même nation, leurs souffrances avaient donné naissance à un esprit d'entraide vite épuisé quand, au début des années cinquante, s'annonça une nette amélioration des conditions de vie. Les films néoréalistes portent la marque de ce bref élan commun.

Entraide et dépassement des conflits sociaux ou idéologiques informent Roma città aperta et Paisà où des personnes qui n'avaient aucune raison de se côtoyer s'unissent et se soutiennent. Les six épisodes de Paisà accompagnent la campagne d'Italie du printemps 1943 à l'hiver 1944. Cinq d'entre eux confrontent des Italiens à des étrangers, surtout Américains. La défiance prévaut d'abord des deux côtés. Peu à peu elle est dépassée, dans la dramatique et très belle étape finale les Américains combattent et meurent avec les partisans. Un épisode particulièrement symbolique, le cinquième, conduit trois chapelains militaires, un catholique, un protestant, un juif, vers un couvent franciscain. S'ils ne portent pas d'armes, les chapelains sont des soldats, ils ont dépassé leurs divergences théologiques quand les moines, juchés sur une colline, loin du champ de bataille, sont perturbés par la venue de deux hérétiques. Le film juxtapose habilement la force, le pragmatisme, la solidité des militaires et la fragilité, la réserve, l'embarras des franciscains, les premiers retrouvent pour un jour une atmosphère de paix qu'ils avaient oubliée, les seconds découvrent l'humanité de ceux pour la conversion desquels ils ont prié. De part et d'autre quelque chose d'indéfinissable s'est produit, tous ont découvert, chez les autres, un monde qu'ils ignoraient.

23 Le conflit, l'occupation provoquent également les rencontres qui scandent Roma città aperta et créent une totale solidarité entre deux hommes que tout devrait séparer, un 
prêtre et un militant communiste. La résistance à l'ennemi, humanitaire et pacifique chez le premier, violente chez le second, les met en rapport. Ils ont chacun leur cercle, le curé sa paroisse où les enfants eux-mêmes participent à la lutte clandestine, le militant ses camarades. Leurs initiatives différentes, animées du même esprit, les amènent à fraterniser dans la prison et dans la mort. L'épilogue du film et celui de Paisà se rejoignent, danger et peine font naître une fraternité entre ceux qui souffrent. Les partisans de Paisà sont des paysans anonymes, aucun ne se distingue à l'écran. Ici rigoureuse, une telle coralità est aussi exceptionnelle, en même temps que Paisà sortait Il sole sorge ancora, premier d'une longue série de films où la résistance apparaît comme une magnifique aventure dont l'un des personnages est le héros.

Une autre coralità se déploie avec Ladri di biciclette où le chômeur, toujours au centre du film, se révèle incapable d'initiative et a toujours besoin d'aide. Nous avons noté comment, dans la première séquence, seule l'amitié d'un camarade lui permet d'obtenir un travail. Sa bicyclette est au mont de piété, il renoncerait à l'emploi si sa femme ne trouvait un moyen de dégager le vélo. Oubliant son engin pendant qu'il pose une affiche, il laisse agir un voleur. Il ne saurait où rechercher la bicyclette si des éboueurs, qui connaissent bien la ville, ne le menaient là où on revend les objets dérobés. Ses camarades partent à l'heure du déjeuner, il serait à nouveau déboussolé sans son fils, gamin d'une dizaine d'années, qui prend les choses en main. Le film, en plus de la déambulation à travers Rome dont nous avons parlé, est une fable sur la faiblesse de l'individu isolé dans un univers dont il ignore les règles. Plusieurs indices suggèrent que, arrivé depuis peu en ville, il se fait mal au milieu urbain. La solidarité, dans cette réalisation qui montre la capitale peu de temps après la fin de la guerre, ne s'oppose pas aux occupants comme dans Roma città aperta ou Paisà, elle répond à la misère ambiante. Errant à travers rues et places le chômeur croise, sans les voir, des travailleurs, des boutiquiers, des revendeurs, des riches et des pauvres, des prostituées, des mendiants. Il cherche son vélo et ne le trouve pas, ses investigations sont vaines parce qu'il n'appartient pas à un groupe qui le soutiendrait jusqu'au bout.

Plusieurs des films dont nous parlons mettent en scène des personnages qui, n'ayant pas ou ayant très peu de rapports avec l'extérieur, vivent en marginaux et ne s'adaptent pas à la société dans laquelle ils se trouvent. Ladri di biciclette, Germania anno zero, Umberto $D$. offrent des images à peu près identiques d'individus isolés, effarés, le regard insensible à ce qui les entoure. Dans Berlin en ruines Edmund, gamin de treize ans, seul pour faire vivre son père malade et son frère recherché par la police, est victime des adultes et des escrocs; Umberto, depuis qu'il a quitté son travail, a perdu tout contact avec collègues et amis. La solitude ici n'a rien de métaphysique, comme ce sera le cas chez Antonioni, elle est sociale et empêche les personnages de se mobiliser, fût-ce pour survivre.

La volonté de coopération, l'esprit d'assistance sont en effet balancés, dans les films néoréalistes, par un sens profond d'une auto-préservation exercée parfois aux dépens des tiers, corale n'est pas synonyme de concorde universelle. Au moment où le chômeur croit avoir trouvé l'individu qui l'a volé, voisins et amis de ce garçon réagissent violemment, l'homme serait lynché si son fils ne réussissait pas à faire intervenir un carabinier; la solidarité s'exerce en faveur d'un familier contre un homme dont le costume, l'accent, les manières montrent qu'il vient d'ailleurs et doit être chassé. À Berlin, la municipalité paie des volontaires pour enterrer les centaines de cadavres victimes des bombardements. Edmund se présente, prend une pelle, commence à 
creuser, la communauté de ceux qui étaient là avant lui, décidée à défendre son privilège, fait bloc et le contraint à partir. À cette union de pauvres contre un plus pauvre La terra trema ajoute une autre forme d'isolement. Le pêcheur qui a soulevé ses camarades contre les mareyeurs décide de se débrouiller par lui-même. Grâce à un prêt gagé par sa maison il achète une barque. Son statut change, il est devenu un patron, admiré mais envié par ses anciens amis, le film qui le montrait au milieu de la foule instaure désormais une distance entre lui et les autres. Une tempête, détruisant la barque, met fin au rêve, la banque saisit la maison, la famille se disloque. Ascension et chute sont également une fable, les pauvres sont trop faibles pour rivaliser individuellement avec les riches, dans leur position la confiance en ses propres forces est vouée à l'échec. Ruiné, le malheureux, fier quand il guidait la révolte ou écoulait sa pêche, semble désormais se recroqueviller et ramper au bas de l'écran.

$\mathrm{Du}$ refus de capituler associant contre l'ennemi, au long de Roma città aperta, des cheminots, des typographes, un prêtre, un militant communiste et un riche antiquaire, de la fraternité construite à travers Paisà, les films néoréalistes sont passés à l'exclusion des étrangers (Ladri di biciclette), à la recherche du succès individuel (La terra trema), au rejet des faibles (Germania anno zero, Umberto D.). En même temps, ils font de l'entraide, de l'action collective, de la capacité à regarder le monde le fondement de toute vie sociale. C'est l'une des leçons d'Umberto $D$., le vieil homme sans espoir se reprend à partir du moment où il a ouvert les yeux sur les autres. Difficile message, le film a été un désastre financier.

\section{Les films devant leur public}

Les films néoréalistes sont une exception dans les années d'après-guerre où drames mondains, comédies, mélodrames constituent l'essentiel de la production italienne. Comment de telles œuvres ont-elles été possibles ? La longue campagne de libération a coûté cher au cinéma, on n'a tourné que vingt et un films en 1944, vingt-huit en 1945, il s'agissait de projets lancés en 1943 et laborieusement terminés. Cinecittà, la cité du cinéma luxueusement équipée par le fascisme a été transformée en camp de réfugiés, la pellicule manque au point qu'on laisse telles quelles les scènes non raccord, les pannes d'électricité interrompent le développement et les films américains, bannis depuis 1938, arrivent par cargaisons entières. Les producteurs n'osent rien entreprendre, les banques, trouvant davantage de profit avec la Reconstruction, ne consentent pas de crédit. La pénurie invite aux solutions originales. Rossellini, très lancé dans le milieu romain, reçoit de trois amis une aide suffisante pour tourner Roma città aperta, et réalise même un tout petit bénéfice, cinq millions de lires réinvestis dans Paisà ${ }^{9}$. Cette fois les contributions amicales ne suffisent pas, Foreign Film production, société anglaise, complète le capital, quarante-quatre millions de rentrées sont réinvestis dans Germania anno zero. Le seul très grand succès du néoréalisme est Ladri di biciclette dont les cent trente millions de bénéfice payent Umberto $D$. Ce film en revanche, tout comme La terra trema ${ }^{10}$, est un échec.

Au tournant des années quarante le néoréalisme ne fait plus recette et ceux qui l'ont animé se replient vers le cinéma à la mode. Les Américains dominent le box-office, ils viennent en tête, en 1951, avec Davide e Betsabea et Quo Vadis?, en 1953 avec La Tunica où se joignent cinémascope, technicolor, son stéréophonique. Les Italiens, pour ne pas se laisser distancer augmentent les budgets, soignent les scénarios, payent les vedettes 
jusqu'à cent millions de lires, construisent des décors monumentaux, s'adaptent à la couleur et à l'écran large. En 1955 treize œuvres italiennes coûtent plus de sept cents millions de lires, le temps des budgets construits à l'aide de dons est définitivement terminé.

Les réactions du public se mesurent donc sur une courte période, 1945-1953. Le néoréalisme devint très tôt un objet de conflit politique, il suscita les passions dans les cercles dirigeants et parmi les intellectuels. L'Église catholique lui fut d'entrée de jeu ouvertement hostile. Depuis 1934 elle s'était dotée d'un "Centre catholique du cinéma » qui publiait, chaque semaine, des Segnalazioni cinematografiche à l'intention des prêtres, des exploitants et des associations familiales. Les jugements qu'on y trouve exprimés sont hautement prévisibles, La terra trema était qualifiée d'œuvre "propre à développer non des sentiments de pitié chrétienne mais des sentiments de haine ${ }^{11}$ ». En dépit de son acharnement, la censure catholique semble toutefois n'avoir exercé qu'une très faible influence; ses attaques contre Roma città aperta réputée « dangereuse pour les enfants » n'ont pas empêché le film d'obtenir un grand succès à Rome où il rappelait à la population les horreurs de l'occupation; peut-être les critiques ont-elles même créé un parfum de scandale et contribué à attirer des curieux. La réaction de l'Église ne doit pas être envisagée par rapport au seul néoréalisme, il faut la considérer dans son ensemble. Empêtrés dans un moralisme rigide, les censeurs catholiques en vinrent à interdire jusqu'aux films américains dont ils appréciaient le conservatisme mais trouvaient les situations "trop osées". À force de s'indigner pour des détails et de critiquer ce qui plaisait au grand nombre, la censure catholique s'est disqualifiée.

31 L'Église pouvait compter sur l'appui du gouvernement, la démocratie chrétienne avait la majorité à la Chambre, contrôlait les ministères et, grâce à la loi d'aide au cinéma, était en mesure de soutenir les seuls films qui lui convenaient ; elle avait également à sa disposition le visa de censure qui permettait de retarder la sortie d'un film ou de l'interdire aux moins de seize ans. Combien de films ont-ils été modifiés par peur de la censure ? Il est impossible de le savoir. Des membres du gouvernement sont intervenus, après le début de la guerre froide, pour exprimer le dégoût que leur inspirait le néoréalisme, mais la seule prise de position nette a été celle d'un sous-secrétaire d'État à la présidence du conseil, Giulio Andreotti qui, tardivement, en février 1952, s'en est pris à Umberto $D$. :

Se è vero che il male si può combattere anche mettendone duramente a nudo gli aspetti più crudi è pur vero che se nel mondo si sarà indotti, erroneamente, a ritenere che quella di Umberto $D$. è l'Italia della metà del secolo ventesimo, De Sica avrà reso un pessimo servigio alla sua patria, che è anche la patria di Don Bosco, del Forlanini e di una progredita legislazione ${ }^{12}$.

Le sens du plaidoyer est clair : que va-t-on penser de nous à l'étranger? Andreotti semblait dire: "Aidez-nous à séduire les étrangers avec des films pleins de soleil ", mais pourquoi n'a-t-il pas lancé cet appel avant 1952 ? D'abord parce que Roma città aperta avait ému beaucoup d'Américains et contribué à modifier l'idée, au départ défavorable, qu'ils se faisaient de l'Italie et aussi parce que, jusqu'à cette date, les films néoréalistes, bien vendus sur les marchés extérieurs avaient été les seuls à rapporter des devises. Rappelons qu'Umberto $D$. fut un échec financier: le pouvoir ne prit ouvertement position que contre un cadavre.

32 La position des communistes semblait beaucoup moins délicate, ils étaient dans l'opposition et ils pouvaient se permettre, tout en combattant l'invasion des écrans par Hollywood, de signaler les films qu'ils estimaient courageux; les attaques de l'Église 
contre De Sica (une bête noire des catholiques, à cause des sanglants portraits de bigots que proposent plusieurs de ses œuvres) leur fournissaient une excellente occasion de dénoncer "l'égocentrisme, les superstitions culturelles et l'enseignement purement académique» autrement dit "la peur des cléricaux, des vieilles perruques, des sénateurs démocrates-chrétiens ${ }^{13} »$.

Rétrospectivement, le parti communiste apparaît comme un allié, ou plutôt comme un ami intéressé du néoréalisme. En 1955, organisant une campagne pour la défense du cinéma italien, L'Unità allait jusqu'à écrire que "l'assaut lancé par les Américains et par nos dirigeants actuels contre le cinéma national italien vise à détruire ce type d'art filmique connu sous le nom de néoréalisme qui a assuré le renom et le succès du cinéma italien à travers le monde entier ${ }^{14} »$. Mais, à cette date, le néoréalisme n'était plus qu'une étiquette et, avant d'en arriver à cette profession de foi, les communistes étaient passés par bien des hésitations. Les réalisateurs auxquels on attribuait l'étiquette "néoréaliste» n'étaient pas des gens commodes: Rossellini, dont l'Église avait peur, se proclamait néanmoins indéfectiblement catholique, ce qui agaçait les hommes de gauche; De Sica protestait avec violence chaque fois que le parti communiste essayait de l'annexer; Visconti était, lui, un vieux compagnon du parti, mais son élégance aristocratique suscitait de très fortes réserves parmi les militants. À cette époque, le parti communiste se sentait profondément mal à l'aise vis à vis du néoréalisme et s'il prenait avec vigueur la défense d'une œuvre que personne ne songeait sérieusement à distribuer dans les circuits commerciaux, La terra trema, sa position ambigüe mérite d'être citée :

Avec ce film tombe la facile étiquette de néoréalisme dont les critiques étrangers avaient affublé notre cinéma pour ses réalisations de l'après-guerre. Il était absurde de parler de néoréalisme avant que n'ait existé un vrai réalisme et celui-ci n'a jamais existé en Italie. Nous pouvons dire de La terra trema qu'il s'agit d'un film réaliste italien ${ }^{15}$.

N'insistons pas sur la pointe de nationalisme qui transparaît ici, elle était de bonne guerre quand on accusait la démocratie chrétienne de succomber à l'Atlantisme.

Les leaders d'opinion reconnus, ceux qui ont orienté la vie publique italienne après la guerre, n'ont exercé qu'une faible influence sur le devenir du cinéma néoréaliste ; les films ont réussi ou échoué selon que le public éprouvait ou n'éprouvait pas l'envie de les voir et les jugements des critiques catholiques ou communistes ont trouvé un très faible écho parmi les spectateurs. Les revues de cinéma, les périodiques littéraires et politiques, s'adressaient à un groupe restreint d'amateurs, les points de vue qui s'y exprimaient reflétaient des conflits internes au monde du cinéma et n'intéressaient pas ceux qui allaient voir des films pour se distraire. Si l'on veut connaître non pas les réactions profondes des Italiens (comment les atteindre?) mais l'information qui leur était couramment proposée, les rotocalchi constituent la seule source utile. Ces hebdomadaires "populaires" ne s'attachaient pas à convaincre leurs clients, ils voulaient seulement leur plaire en flattant leurs préférences, ce qu'ils imprimaient correspondait à ce qu'ils croyaient être l'avis des lecteurs. Même s'ils se trompaient, ils étaient sûrement plus proches du sentiment général que les journaux politiques ou les magazines filmiques.

Prenons les deux hebdomadaires qui ont connu la meilleure diffusion entre 1946 et 1952, La Domenica del Corriere et Tempo ${ }^{16}$. Discrets l'un comme l'autre sur les affaires politiques, les deux hebdomadaires s'intéressaient aux voyages, à l'économie, aux questions familiales, au sport, aux spectacles et en particulier à la production filmique. 
Les articles sur le cinéma, en général bien illustrés, étaient rapides mais précis, ils abordaient des questions rarement traitées, parlaient des films danois ou mexicains, donnaient quelques détails sur l'économie et la production cinématographiques, offraient des résumés de films clairs et vivants. Leurs jugements témoignaient d'une large ouverture d'esprit; Orson Welles, était-il dit dès 1947, « est l'un des scénaristes, metteurs en scène et acteurs les plus inspirés qui se soient mis au service du cinéma $^{17} »$ : peu de critiques, à l'époque, savaient que Welles, réalisateur, était également scénariste. Hollywood avait droit à de longs commentaires, le star system était dénoncé comme dommageable pour les acteurs qu'il obligeait à soigner autant leur image à la ville que leur travail de scène; les Européens n'étaient pas oubliés, consacrer une demi-page à James Mason en $1947^{18}$ manifestait une bonne connaissance des jeunes talents.

Dans ces hebdomadaires, le cinéma italien n'était ni ignoré, ni surestimé. Évidemment, le terme "néoréalisme " n'était jamais employé. Néanmoins ce que nous désignons maintenant sous cette étiquette était doublement présent. D'abord, les succès internationaux obtenus par Rossellini et De Sica étaient un objet de permanente fierté ; Tempo, prenant acte de jugements favorables portés par des journaux américains, rappelait que Sciuscià était «un des plus forts cris de désespoir lancés dans notre siècle » et ajoutait aussitôt que, avec des œuvres d'une telle densité, « l'Italie apparaît comme la seule nation qui soit capable de produire des films intelligents et courageux ${ }^{19} »$. Mais l'influence exercée par ces deux cinéastes ne se résumait pas aux commentaires nombreux, enthousiastes qu'on dédiait à leurs films, leur marque se retrouve dans de nombreux reportages ayant trait à la vie sociale; ainsi La Domenica consacrait-t-elle ${ }^{20}$ une page entière, illustrée de six clichés, à un camp pour orphelins où tout était fait pour montrer que le message de Sciuscià avait été entendu; ici et dans d'autres articles l'illustration était conçue en référence au cinéma dont les photographes s'attachaient à restituer les cadrages, et même parfois le contenu.

J'ai choisi à dessein l'exemple de Sciuscià dont la carrière avait été désastreuse dans la Péninsule : les magazines ne se laissaient pas impressionner par le faible nombre des entrées, ils distinguaient nettement les réactions de l'étranger et celles du public local ; loin de voir dans le film une image négative propre à salir la réputation des Italiens, ils étaient persuadés, en 1948, que le cinéma pouvait à la fois alerter l'opinion internationale, surtout américaine, sur les difficultés de l'Italie et faire avancer la recherche de solutions dans le pays. On comprend ainsi mieux pourquoi le gouvernement, malgré l'exaspération qu'il ressentait, et malgré les pressions de la hiérarchie catholique, n'osa pas s'en prendre au cinéma avant 1952. Quand Andreotti se décida à publier son très sévère article, le néoréalisme n'avait plus beaucoup d'importance.

\section{Exégèse d'un néologisme}

Le terme « néoréalisme » n'était pas encore en usage qu'on débattait déjà âprement sur le caractère innovant de Roma città aperta et Paisà. Depuis, la bibliographie du néoréalisme cinématographique est devenue gigantesque, il faudrait un article bien plus long que celui-ci pour en rendre compte de manière exhaustive. Cet effort aurait-il un sens? Beaucoup de textes sont l'expression d'une humeur passagère et les perspectives sur le cinéma d'après-guerre ont beaucoup évolué pendant les dernières 
décennies grâce aux chercheurs italiens et américains, dans une moindre mesure anglais et allemands. Deux ouvrages récents, Il cinema neorealista italiano. Storia economica, politica e culturale de Gian Piero Brunetta ${ }^{21}$ et Neorealismo. Il nuovo cinema del dopoguerra de Stefania Parigi ${ }^{22}$ font la synthèse de ce que l'on peut savoir aujourd'hui. Les deux ouvrages considèrent le néoréalisme sous un angle extrêmement large et classent les films sous le nom de leur réalisateur. Historien du cinéma mondialement connu, Gian Piero Brunetta offre une étude systématique où il prend en compte le contexte social et intellectuel (il y eut également un néoréalisme littéraire), le passé et les objectifs des réalisateurs, les conditions de production et de réception, l'accueil fait aux œuvres. Stefania Parigi se montre moins analytique et plus ambitieuse. S'interrogeant sur les sens possibles d'une expression devenue universelle sans avoir été jamais définie et sur la cohérence idéologique reliant des films qui se réfèrent à des valeurs incompatibles, au christianisme, au marxisme, au libéralisme, elle souligne, entre ces œuvres, une concordance " humaniste ", une commune référence à la dignité de l'être humain, la persistance « di una condizione umana fondata, in tutti i casi, sulla sofferenza e sulle rovine ".

Dans ce panorama, la discrétion de la France paraît singulière. Ce sont pourtant deux critiques français, le communiste Georges Sadoul, le chrétien André Bazin qui, dès 1945, ont milité activement dans la presse, les festivals, les ciné-clubs pour faire reconnaître l'importance des films italiens et conduit le public français, d'abord médiocrement intéressé, à reconnaître l'apport du néoréalisme. Dans L'écran français, populaire hebdomadaire cinématographique, Sadoul répéta de semaine en semaine qu'un «nouveau cinéma » était en train de voir le jour, ses chroniques et ses interviews popularisèrent les cinéastes italiens en France et convainquirent les dirigeants du PCF que des films comme Ladri di biciclette et La terra trema étaient des armes pour la lutte sociale.

Plus théoricien, André Bazin mourut très jeune avant d'avoir mis en train le traité du cinéma dont il rêvait, son œuvre se compose essentiellement d'articles de circonstance. Perspicaces, sensibles, ses textes consacrés au néoréalisme, réunis à la fin du volume qu'il avait intitulé $Q u^{\prime} e s t-c e$ que le cinéma ${ }^{23}$, suivent l'actualité et ne proposent pas une vue d'ensemble du sujet. En 1948, il parle de "l'école italienne de la libération ", l'année suivante il met néoréalisme entre guillemets, puis il adopte définitivement le mot. Ladri di biciclette l'enthousiasme d'emblée comme « reportage reconstitué » doté " d'une valeur documentaire exceptionnelle » par son " adhérence parfaite et naturelle à l'actualité » et « une adhésion spirituelle à l'époque ${ }^{24}$ ». Le réalisme est ici pris au pied de la lettre, il est l'observation du monde comme il va. L'approche change et se nuance avec La terra trema, la proximité aux êtres et aux choses, reconnaît Bazin, n'est pas une simple observation, «le naturel suprême, ce sentiment d'événements observés par hasard au fil des heures est le résultat de tout un système esthétique présent (quoique invisible ${ }^{25}$ » - un «système » ramené à l'absence d'acteurs, l'absence de mise en scène (?) et l'absence d'histoire dans la mesure où les scènes au lieu de se commander les unes les autres, font place à une «succession des instants de la vie dont aucun ne peut être dit plus important que les autres ${ }^{26} »$. Peu de critiques, à l'époque, avaient aussi bien mis en relief la minceur des intrigues et le caractère incertain, aléatoire de la logique narrative. En revanche, la mise en scène minutieusement organisée de La terra trema, le soin apporté aux éclairages, aux cadrages sont curieusement oubliés, l'idée d'une intense libération, d'une sorte d'anonymat de la réalisation, parfois citée comme 
caractéristique du néoréalisme conduit ici à une surévaluation de "l'absence » que Bazin aurait sûrement corrigée s'il avait écrit son grand ouvrage.

\section{Ce que retient le $x \mathrm{XI}^{\mathrm{e}}$ siècle}

41 Passé Bazin : le vide, l'édition française sort deux ouvrages en tout et pour tout ${ }^{27}$. Le néoréalisme italien et ses créateurs de Patrice Hovald ${ }^{28}$, Le néo-réalisme italien, une expérience de cinéma social de Raymond Borde et André Bouissy ${ }^{29}$ n'ont d'intérêt que rétrospectivement, ils représentent les courants d'interprétation alors dominant, chrétien, idéaliste, sensible et doloriste pour le premier, marxisant, progressiste, militant pour le second.

Le regard porté sur les films varie en fonction des circonstances historiques et des canons esthétiques. En 1950 le cinéma apparaissait comme un divertissement et un moyen de découvrir un monde immense et mal connu. Les œuvres qualifiées de néoréalistes intéressaient par leur intrigue touchante et par les aperçus qu'elles ouvraient sur une Italie, une Europe en ruines. Les drames évoqués, guerre, massacres, torture, chômage, abandon d'enfants, indifférence au sort des vieillards, exploitation des faibles sont encore les nôtres, mais le contexte a changé, le monde s'est rétréci, l'Europe y est devenue une modeste péninsule. Interfaces entre les individus et leur entourage, des écrans, installés partout, ont enlevé aux films leur rôle d'informateurs visuels; le cinéma distrait, il ne découvre plus un univers que captent et reflètent beaucoup mieux d'autres vecteurs de communication.

Les «vieux» films attendrissent comme le font les photographies anciennes, on les regarde pour mesurer les énormes transformations intervenues en peu d'années, les œuvres néoréalistes ont, à peu près seules, le rare privilège d'apprendre quelque chose aux réalisateurs et de plaire encore aux cinéphiles. Leur dénonciation des injustices et des inégalités, leur pessimisme, percutants à l'époque où ils sont sortis, paraissent timides et trop étroitement européens. En revanche, leur capacité à soutenir différentes lectures impressionne toujours. Le personnage central n'y est pas un héros auquel on tendrait à s'identifier, on le suit, on ne se passionne pas pour lui; l'entourage, le groupe, les soutiens et les adversaires retiennent autant que lui l'attention, le spectateur ne se sent pas conduit par un enchaînement serré d'événements, il s'abstrait parfois de l'intrigue pour s'attacher au défilement des images, à une déambulation imprévisible que ne justifient pas les aventures d'un personnage central. Plus spectaculaire que narratif, privilégiant les effets spéciaux, le mouvement, le franchissement de l'espace par rapport à l'histoire et aux individualités, le cinéma contemporain est davantage proche du néoréalisme que du cinéma classique hollywoodien. Le néoréalisme avait tenté d'arracher le cinéma à la seule linéarité narrative, le cinéma d'aujourd'hui, sans chercher une impossible ressemblance, y trouve comme un prélude à sa propre évolution. 


\section{NOTES}

1. Bianco e Nero, vol. XI, 4 avril 1950.

2. 1946, scénario: Zavattini, Antonioni, Barbaro; réalisation: Giuseppe De Santis; chef opérateur : Otello Martelli.

3. 1948, scénario: Alvaro, Lizzani ; réalisation: Giuseppe De Santis; chef opérateur: Otello Martelli.

4. 1950, scénario : Fellini, Pinelli ; réalisation : Pietro Germi ; chef opérateur : Leonardo Barboni.

5. Cinema, vol. VIII, 15 février 1949.

6. La fin n'est pas sans rappeler Duel in the Sun, film américain sorti l'année précédente. La référence à Hollywood, implicite ici, est patente dans Il cammino della speranza dont une partie est un simple démarcage du film américain The Grapes of Wrath.

7. En se référant aux Malavoglia de Giovanni Verga le film souligne d'ailleurs la permanence de cette exploitation.

8. Bianco e Nero, vol. X, 7 juillet 1949.

9. Un correctif est nécessaire à propos du succès en salle de ce film. Le distributeur déçu revendit le film qui, à partir de sa projection à New York, rapporta quatre fois ce qu'il avait coûté.

10. La terra trema est cependant un cas particulier, rien n'a été fait pour en assurer le succès. La préparation avec les pêcheurs a duré plus d'un an, le producteur a changé en cours de route, le film dure deux heures quarante, longueur exceptionnelle à l'époque, les personnages parlent un dialecte incompréhensible à la majorité des Italiens, il a fallu lui ajouter des sous-titres.

11. Segnalazioni cinematografiche, 1948, vol. XXIV, $n^{\circ} 15$, p. 119.

12. Libertas, 24 février 1952.

13. L'Unità, 5 décembre 1948.

14. L'Unità, 20 mai 1955.

15. L'Unità, 4 septembre 1948.

16. J'ai laissé de côté Epoca dont la publication ne commence qu'en 1950.

17. Domenica del Corriere, 30 mars 1947, p. 9.

18. Tempo, 23 mars 1947.

19. 15 novembre 1947, p. 14.

20. 9 mars 1947, p. 5.

21. Rome, Laterza, 2009.

22. Venise, Marsilio, 2014.

23. D'abord publié en quatre fascicules entre 1958 et 1962, réunis ensuite en un volume, Paris, Éditions du Cerf, 1975.

24. A. Bazin, Qu'est-ce que le cinéma?, ouvr. cité, p. 263. L'article intitulé «Le réalisme cinématographique et l'école italienne de la libération » est paru initialement dans Esprit, $\mathrm{n}^{\circ} 141$, janvier 1948, p. 58-83.

25. A. Bazin, Qu'est-ce que le cinéma ?, ouvr. cité, p. 305. L'article intitulé « Le Voleur de bicyclette, ou l'épreuve victorieuse du néo-réalisme italien" est paru initialement dans Esprit, $\mathrm{n}^{\circ} 161$, novembre 1949, p. 820-832.

26. Ibid., p. 333.

27. Pour être complet mentionnons deux numéros de revues, Le Néo-réalisme italien, Études cinématographiques, 1964 ; Le Néoréalisme italien, Cinémaction, 1994. Comme il est de règle dans ce genre de publication les textes, inégaux, traitent des points mineurs et n'analysent pas le néoréalisme.

28. Paris, Éditions du Cerf, 1959.

29. Lausanne, Éditions de la Cinémathèque suisse, 1960. 


\section{RÉSUMÉS}

Le néoréalisme est, dans la culture italienne comme dans la tradition cinématographique mondiale, une pierre milliaire, à la fois ensemble d'œuvres exceptionnelles et modèle stylistique. L'expérience fut pourtant brève, à peine sept ans, et le nombre de films à proprement parler «néoréalistes » très limité. Dans la période pleine d'incertitudes qui suivit la fin de la guerre, les cinéastes, libérés des règles et des obligations imposées par les producteurs, ont tourné sans se préoccuper de morale, de logique narrative, de conclusions heureuses. La reconstruction terminée, les banques rétablirent leur contrôle sur les studios, les forçant à relancer le « cinéma classique ». La mémoire du bref épisode néoréaliste est restée comme exemple de ce qui pourrait être un cinéma en liberté.

Nella cultura italiana e nella tradizione cinematografica mondiale, il neorealismo costituisce una pietra miliare, sia per la qualità eccezionale delle opere che come modello stilistico. Pure, l'esperienza è stata breve, appena sette anni, e il numero di film propriamente «neorealisti» è molto limitato. Nel periodo pieno d'incertezze che seguì la fine della guerra i cineasti, liberi delle regole e degli obblighi imposti dai produttori, girarono i loro film senza preoccuparsi di moralismo, di logica narrativa, di scioglimenti felici. Finita la ricostruzione, le banche ristabilirono il controllo sugli studi, costringendoli a rilanciare il «cinema classico». La memoria del breve episodio neorealista è rimasta come esempio di quello che potrebbe essere un cinema in libertà.

\section{INDEX}

Mots-clés : histoire du cinéma, cinéphilie, néoréalisme, résistance, censure

Parole chiave : storia del cinema, cinefilia, neorealismo, resistenza, censura

\section{AUTEUR}

\section{PIERRE SORLIN}

Université Sorbonne Nouvelle - Paris 3.

pierre.sorlin@orange.fr

Pierre Sorlin est professeur émérite à l'Institut d'études et de recherches cinématographiques et audiovisuelles de l'université Paris 3. Ses recherches portent, entre autres, sur l'histoire et la sociologie du cinéma. 